Review Article

\title{
ROLE OF PHYTOCHEMICALS IN ORAL POTENTIALLY MALIGNANT DISORDERS : A REVIEW
}

\author{
Ananya Madiyal', Vidya Ajila², Subhas G Babu', Shruthi Hedge, Harini Keshavaiah ${ }^{5}$ \\ \& Priyadharshini Alva $\mathrm{M}^{6}$ \\ ${ }^{1,6}$ Post Graduates, ${ }^{2}$ Reader, ${ }^{3}$ Professor $\&$ HOD,, 4.5 Lecturers, Department of Oral Medicine $\&$ Radiology, ${ }^{5}$ Department of \\ Periodontics, A.B. Shetty M emorial Institute of Dental Sciences, Nitte University, M angalore - 575 018, India.
}

Correspondence :
Ananya Madiyal

Post Graduate, Department of Oral M edicine and Radiology, A.B. Shetty Memorial Institute of Dental Sciences Nitte University, M angalore - 575 018, India.

Mobile : +9195388 40189 E-mail : ananyamadiyal@gmail.com

\begin{abstract}
:
Oral cancer is a major global health concern and poses a challenge to diagnostic and therapeutic aspects of healthcare services. Various oral lesions like leukoplakia, erythroplakia, lichen planus and oral submucus fibrosis categorized as orally potential malignant disorders have shown increased incidence of malignant transformation. Free radicals are highly reactive chemical species with capacity to damage nucleic acids, proteins and lipids and bring about changes of clinical significance. Antioxidants help in scavenging these free radicals and prevent disease progression. Naturally occurring phytochemicals play an important role in preventing oxidative stress and protect the cells from damage by free radicals.
\end{abstract}

Keywords: Antioxidants, phytochemicals, oral potentially malignant disorders, chemoprevention

\section{Introduction:}

Oral cancer is a major global health concern with increasing prevalence and high levels of mortality. It poses a major challenge to diagnostic and therapeutic aspects of the healthcare services. Oral and pharyngeal cancers, grouped together constitute the sixth most common cancer in the world. ${ }^{1}$ The annual estimated incidence of oral cancer stands at about 275,000 and pharyngeal cancer at about 130,300 , excluding nasopharyngeal cancers in developing countries. ${ }^{2}$ The areas characterised by high incidence rates for oral cancer include South and South-East Asia ( Sri Lanka, India, Taiwan and Pakistan), parts of western ( France) and Eastern Europe ( Hungary, Slovakia and Slovenia), parts of Latin America and the Caribbean (Brazil, Uruguay and Puerto Rico) and in the Pacific regions (Papua

\begin{tabular}{|c|}
\hline Access this article online \\
\hline Quick Response Code \\
\hline
\end{tabular}
$\mathrm{N}$ e w Guinea and Melanesia) ${ }^{1}$ Oral cancer is one of the most common forms of malignancies in India with a prevalence varying from $11 \%$ to $52 \%$ of all diagnosed cancers in the country. ${ }^{3}$ National Cancer Registry, 2003 identified that the spectrum of the diseases in the country lies in states like Uttar Pradesh, Madhya Pradesh, Bihar, Maharashtra, Gujarat, Andra Pradesh, Karnataka and Tamil Nadu. Variation in incidence depends upon geographic location and adverse habits.

Prognosis of oral cancer is generally poor with a five year survival rate of less than $50 \%{ }^{4}$ Local recurrences as well as lymph node metastasis occur in a significant number of the affected people while distant metastasis is often rare. Various oral lesions like leukoplakia, erythroplakia, lichen planus and oral submucous fibrosis are known to have an increased risk of progressing into malignancy. These lesions are collectively termed as 'oral potentially malignant disorders'. WHO in 2005 defined oral potentially malignant disorder as 'the risk of cancer being present in a pre-cancerous lesion or condition, either at the time of initial diagnosis or in the future ${ }^{15}$ The term conveys that not all of the lesions described under it will turn into cancer but instead, it blankets a family of morphologic alterations that will potentiate some of the lesions into a malignant transformation. WHO first classified oral potentially 
malignant disorders as potentially malignant lesions and potentially malignant conditions (table 1 ) $^{6}$ in 1978. A classification based on eitiology of the lesion was later given by Anthony George et al in 2011 (table 2)

It is a well-established fact that virtually all oral cancers are preceded by visible clinical changes in the oral mucosa usually in the form of red or white patches. ${ }^{8}$ Early diagnosis of these potentially malignant disorders help in avoiding high levels of morbidity and mortality associated with these lesions. Oxidative stress has always been associated with the development of a wide variety of diseases. It is a process derived from the inability of the body's endogenous antioxidant defences to scavenge free radicals. Free radicals are highly reactive chemical species characterized by short half-life and are made up of a single atom or groups of atoms that form a molecule with a free electron. ${ }^{9}$ This electron is responsible for the high reactivity of free radicals. When free radical derived oxidative damage to nucleic acid, proteins and lipids of extra cellular and cellular matrix is observed, it produces damages of clinical importance. Dietary antioxidants can reduce the risk of cancer. Phenolic compounds derived from a variety of fruits and vegetables show anti-inflammatory and anticancer effect. Supplements of phytochemicals including carotenoids and flavonoids demonstrate chemopreventive and chemoprotective activity especially in the cellular proliferation phase.

\section{Rationale behind the use of antioxidants in management of oral potentially malignant disorders}

Generation of reactive oxygen species by biologic systems either by normal metabolic pathways or as a consequence of exposure to chemical carcinogens contributes to the multi-stage process of carcinogenesis. ${ }^{10}$ Some examples of antioxidants include Vitamin C (Ascorbic acid), Vitamin E (Alpha tocopherol), retinol, retinoids and beta-carotene. In 1981 it was discovered that there was an association between low serum beta carotene levels and cancer. ${ }^{11}$ There exists an inverse relationship between the levels of these antioxidants in the diet and in the serum and the probability of development of cancer. ${ }^{12}$ Cancer develops due to genetic predisposition or due to irreparable oxidative damages to the DNA that cause mutations. Antioxidants are essential in reducing this oxidative stress by scavenging free radicals and thereby reducing mutations, changes in enzyme activity and lipid peroxidation of cellular elements.

Cancer therapeutics mainly concentrates on the final stage of the disease. This limits its preventive potential. Refocus of management of cancer from the end point to the progression of the disease is essential as these interventions will prevent, inhibit and reverse the process of carcinogenesis thereby reducing the long term effects on such individuals. Early detection and treatment of oral potentially malignant disorders will provide a better standard of life to individuals affected by oral cancers.

\section{Phytochemicals}

The word phytochemicals is derived from the Greek word 'Phyto' meaning plant. More than 5000 different types of phytochemicals have been identified but they remain just a small percentage of the total number of such plant chemicals. Phytochemicals are defined as 'bioactive nonnutrient plant compounds in fruits, vegetables, grains and other plant foods that have been linked to reducing the risk of major chronic diseases.' ${ }^{13}$

Some of the major phytochemicals are:

\section{Beta-carotene}

Approximately $16 \%$ of the ingested beta-carotene is transformed to retinol by a two-step process in the intestinal mucosa. It is absorbed by the lymphatics and is not taken up by the extra hepatic tissues like bone marrow, blood cells, spleen, adipose tissue, lungs, muscles and kidney ${ }^{14}$ The possible protective effects of beta carotene is attributed to its antioxidant action by trapping free radicals, particularly peroxyl and hydroxyl which are involved in aging and carcinogenesis. It also increases cell mediated immune response due to increased monocyte expression and increased response of tumour necrosis factor alpha. Lower than expected levels of serum beta carotene have been seen in men who smoke cigarettes and 
consume alcohol. Low levels of beta carotene is associated with increased risk of oral cancer. ${ }^{11} \mathrm{An}$ abundance of betacarotene has been found in a variety of yellow and green fruits and vegetables. ${ }^{13}$

\section{Lycopene}

Lycopene is one of the most potent antioxidant and has been hypothesised to prevent carcinogenesis and atherogenesis by protecting critical biomolecules like lipids, lipoproteins and DNA. ${ }^{15}$ Lycopene suppresses carcinogen induced phosphorylation of regulatory proteins such as p53 and Rb anti-oncogenes and stops cell division at $\mathrm{G}_{0}-\mathrm{G}_{1}$ cell cycle phase. ${ }^{16}$ It also reduces cellular proliferation induced by potent mitogens like insulin-like growth factors in various cancer cell lines. ${ }^{17}$ It exhibits highest physical quenching rate constant with singlet oxygen. Lycopene is a bright red pigment found in tomatoes and other red fruits like red carrots, watermelons and papayas. ${ }^{18}$

\section{Flavonoids}

Flavonoids are a group of plant derived phenolic compounds that have been identified to have antioxidant property to reduce the risk of development of chronic diseases. Flavonoids have an additive effect to the endogenous scavenging systems ${ }^{19}$.

They have different functions in the antioxidant system like:

\section{a. Direct Radical Scavenging}

Flavonoids stabilize the reactive oxygen species by reacting with the reactive compound of the radical thereby producing a less reactive radical. Few flavonoids directly scavenge superoxide while others scavenge peroxynitrites. $^{20}$

\section{b. Nitric Oxide}

Nitric oxide produced by macrophages reacts with free radicals producing peroxynitrite which can react with low density lipoproteins thereby producing irreversible injury to the cell membrane. Flavonoids scavenge nitric oxide as well as peroxynitrite thus reducing the amount of oxidative damage. ${ }^{21}$

\section{c. Xanthine Oxidase}

Xanthine dehydrogenase, an enzyme present under physiologic conditions is changed to xanthine oxidase during ischemic conditions. During reperfusion, xanthine oxidase reacts with molecular oxygen thereby producing superoxide free radicals. Flavonoids scavenge these superoxide molecules thus resulting in less oxidative injury. ${ }^{22}$

\section{d. Leukocyte Immobilization}

During ischemia and inflammation, leukocytes which are freely moving along the endothelial wall are acted upon by endothelium derived mediators and complement factors and adhere to the endothelial wall and stimulate degranulation of neutrophils. Flavonoids cause a decrease in the number of immobilized leukocytes and total serum complement. They also cause a decrease in the degranulation of neutrophils and mast cells and provide a protective action during reperfusion by modulation of receptor directed calcium channels in the plasma membrane. ${ }^{23}$

One of the best described group of flavonoids, quercetin is found in abundance in onions, apples, broccoli and berries. The second group, narigin is found in citrus fruits. Flavonoids belonging to the catechin group are found primarily in green and black tea as well as in red wine. The last group of flavonoids, anthocyanins are found in strawberries, grapes, wine and tea. ${ }^{18,24}$

\section{Role of phytochemicals in the prevention of cancer}

Free radicals are constantly produced in the body as a result of normal metabolic processes. The key to maintaining health is achieving a balance between free radicals and antioxidants. Over production of free radicals causes oxidative damage to cell membrane lipids, proteins and DNA leading to cancer production. To prevent or slow down the oxidative stress induced by free radicals, sufficient amounts of antioxidants should be consumed. Phytochemicals like carotenoids and flavonoids help to prevent cellular systems from oxidative stress and may also lower the risk of chronic disease. ${ }^{13}$ Phytochemicals have a complementary and overlapping mechanism of action like 
scavenging of free radicals, regulation of gene expression in cell proliferation and differentiation, inhibition of expression of oncogenes, induction of tumor suppressor gene expression, modulation of enzyme activity in detoxification and regulation of hormone metabolism. ${ }^{25,26}$

Natural phytochemicals present in fruits and vegetables have strong antioxidant and anti-proliferative activities. The synergistic effect of various phytochemicals is responsible for its beneficial effect. Individual antioxidants produce varying degrees of tumor regression only at very high doses which is frequently associated with toxicity. At lower doses they may be ineffective or stimulate the growth of tumour cells. ${ }^{27}$ Therefore the use of a single antioxidant has no therapeutic or clinical significance. Protection against free radicals can be enhanced by consuming a mixture of dietary antioxidants thereby avoiding the toxicity associated with high doses or growth stimulation associated with low doses.

\section{Antioxidantsin therapeutics of oral lesions}

In general, pre-malignant lesions are not lethal by themselves and are associated with low risk of malignant transformation. The goal of cancer therapeutics is not only treatment of cancer but also the suppression and reversal of pre-malignant lesions. The possible use of antioxidants in oral mucosal lesions include the following: ${ }^{27}$

1. Prevention of lesions in high risk individuals with mucosa that clinically appears normal with no history of pre-malignant or malignant lesion.

2. Treatment of pre-malignant oral lesions.

3. In patients who had pre-malignant or malignant lesions that have been successfully treated, in order to prevent recurrence of the treated initial lesion or to prevent the development of a secondary or a separate primary.

Non-surgical management of pre-cancerous lesions should be considered, especially if they involve a large surface area and in medically compromised patients. It also provides additional advantages of being non-invasive, relatively cost effective and ease of application. Patients with dermatologic disorders receive doses of beta-carotene as high as $300 \mathrm{mg}$ per day for years with only carotenodermia as a side effect. Clinical trials use doses considerably less than $300 \mathrm{mg}$ per day as beta-carotene levels are minimally elevated when the dose is substantially increased and the possibility of carotenodermia is reduced. ${ }^{28}$ There was absence of side effects in patients supplemented with 26 $120 \mathrm{mg}$ of beta-carotene per day. ${ }^{29} \mathrm{~A}$ combination of betacarotene with vitamin A or retinoids has showed some success in the treatment of oral leukoplakia. ${ }^{30}$ Alphatocopherol in doses as high as $3200 \mathrm{mg}$ per day have been well tolerated by adults without any signs of toxicity. ${ }^{31}$ Supplements are more effective in achieving high plasma alpha-tocopherol levels than diet modifications. It plays an important role in protecting the cell membranes from lipid peroxidation. Administration of $800 \mathrm{IU}$ of alpha-tocopherol per day for 24 weeks shows some clinical response in patients with oral potentially malignant disorders. ${ }^{31} 16 \mathrm{mg}$ of lycopene in divided doses has shown improvement in mouth opening and burning sensation in patients with oral submucous fibrosis. ${ }^{18}$ Oral supplementation of 4-8 mg of lycopene given over a period of eight months showed significant reduction in hyperkeratosis in patients with oral leukoplakia with no clinical signs of toxicity. ${ }^{32}$

A practical strategy for people who want to optimize their health through diet modification is by increasing the consumption of fruits, vegetables and whole grains that are rich in antioxidants. A combination of orange, apple, grape and blueberry has displayed a synergistic effect in delivering these antioxidants in sufficient quantities. The phytochemicals present in food differ in their molecular size, polarity and solubility which may affect their bioavailability and distribution in various macromolecules, subcellular organelles, cells, organs and tissues. This synergistic and additive effect of whole foods has been proposed to be responsible for their potent antioxidant and anticancer effects. ${ }^{13}$

\section{Summary :}

Normal metabolic processes produce free radicals in the body. This mechanism is accentuated in the presence of precipitating factors such as smoking or alcohol 
Table 1

\begin{tabular}{|l|l|}
\hline Potentially Malignant Disorders & Potentially M alignant Lesions \\
\hline Oral submucous fibrosis & Leukoplakia \\
\hline Actinic keratosis & Erythroplakia \\
\hline Lichen planus & Palatal lesions in reverse smokers \\
\hline Discoid lupus erythematosus & \\
\hline
\end{tabular}

Table 2

\begin{tabular}{|l|l|l|l|l|}
\hline High risk & Lifestyle related & Infections & Immunodeficieny & Inherited disorders \\
\hline Leukoplakia & Smokeless tobacco keratosis & $\begin{array}{l}\text { Hyperplastic } \\
\text { candidiasis }\end{array}$ & $\begin{array}{l}\text { Solid organ } \\
\text { transplantation }\end{array}$ & $\begin{array}{l}\text { Xeroderma } \\
\text { pigmentosa }\end{array}$ \\
\hline Erythroplakia & Reverse smoker's palate & $\begin{array}{l}\text { Viral (HPV, HIV, } \\
\text { EBV, HBV, HSV) }\end{array}$ & $\begin{array}{l}\text { Graft versus host } \\
\text { disease }\end{array}$ & $\begin{array}{l}\text { Dyskeratosis } \\
\text { congenita }\end{array}$ \\
\hline Oral submucous fibrosis & Actinic chelitis & Tertiary syphilis & $\begin{array}{l}\text { Chronic cutaneous } \\
\text { lupus erythematosus }\end{array}$ & $\begin{array}{l}\text { Epidermolysis } \\
\text { bullosa }\end{array}$ \\
\hline Erosive lichen planus & & & & Bloom syndrome \\
\hline & & & & Fanconi's anemia \\
\hline
\end{tabular}

consumption. This causes generation of excess of free radicals. A balance between oxidative free radicals and antioxidants is maintained in the body by oxygen scavengers. In cases where there is an excess of free radical production, these scavenging systems are overwhelmed and fail to reduce the oxidative stress. This causes damage to the cell membrane phospholipids and cell organelles and is termed as oxidative damage. Pre-cancer, cancer and other chronic diseases are mainly caused as a result of this oxidative damage. Use of antioxidant supplements shows promise in the resolution of such potentially malignant disorders.

Phytochemicals show a synergistic function when supplemented along with other antioxidants. Use of a single antioxidant at levels that can suppress or cause regression of pre-malignant lesions may produce features of toxicity like carotenodermia and hypervitaminosis. Hence a combination of various antioxidants should be used for the treatment of such disorders. Before making the decision to use antioxidants for treatment, it is critical to obtain histopathologic diagnosis of the lesion. Lesions diagnosed as hyperkeratotic will take considerable amount of time before showing clinical signs of improvement while lesions diagnosed as dysplastic may not show any positive change. Newer analogues of synthetic retinoic acid show better efficacy of treatment with less instances of toxicity. While surgical management remains the mainstay of treatment of oral potentially malignant disorders, use of antioxidants, particularly newer analogues are gaining importance as non-invasive treatment options. Chemoprevention with these agents is useful in advanced disease or in an adjuvant setting in combination with surgical management, steroid hormones and cytotoxic chemotherapy that are currently available as treatment for patients with oral potentially malignant disorders.

No single antioxidant can replace the health benefits of a diet with a complex mixture of phytochemicals derived from a combination of various fruits, vegetables and whole grains. Phytochemical extracts from such fruits and vegetables have strong antioxidant and antiproliferative effects and a major part of the total antioxidant activity is from the combination of these phytochemicals. Diet modifications along with dietary supplements, neutraceuticals and functional foods can help prevent the development and progression of diseases like cancer. Further research on the health benefits, efficacy and safety of phytochemicals in whole foods is warranted. 


\section{References :}

1. Warnakulasuriya S. Global epidemiology of oral and pharyngeal cancer. Oral Oncology 2009; 45: 309-16.

2. FerleyJ, Parkin DM . GLOBOCAN 2002. Cancer incidence, M ortality and Prevalence Worldwide. IARC Cancer Base (2002 estimates). Lyon IARC press 2004.

3. Tuyns AJ, Hiramaya T. World Health Organisation Epidemiologic Studies of the Tumors of the M outh and Jaw. J Dent Res 1966; 45: 53545.

4. M elhotra R, Pandya S, Chaudary AK, Kumar M, Singh M. Prevalence of Oral Pre-malignant and M alignant Lesions at a Tertiary Level Hospital in Allahabad, India. Asian Pacific] Cancer Prev 2008; 9: 263-6.

5. Warnakulasuriya S, Johnson NW, van der Waal I. Nomenclature and Classification of Potentially M alignant Disorders of the Oral M ucosa. J Oral Pathol Med 2007; 36: 575-80.

6. World Health Organization Collaborating Centre for Oral Precancerous Lesions. Definition of leukoplakia and related lesions: an aid to studies on oral precancer. Oral Surg Oral M ed Oral Pathol1978; 46: 518-39.

7. George A, Sreenivasan B, Sunil S, Varghese S, Thomas], Gopakumar D, Varghese M. Potentially Malignant Disorders of Oral Cavity. OM PJ 2011; 2: 95-100.

8. Reibel J. Prognosis of Oral Pre-malignant lesions: Significance of Clinical, Histopathological and Molecular Biological Characteristics. Crit Rev Oral Biol M ed 2003; 14: 47-62.

9. Iannitti T, Rottigni V, Palmieri B. Role of Free Radicals and Antioxidant Defences in Oral Cavity-Related Pathologies. J Oral Pathol M ed 2012; 649-61.

10. Khan SG, Katiyar SK, Agarwal R, Mukhtar H. Enhancement of Antioxidant and Phase II Enzymes by Oral Feeding of Green Tea Polyphenols in Drinking Water to SKH-1 Hairless M ice: Possible Role in Cancer Chemoprevention. Cancer Res 1992; 52: 4050-2.

11. Peto R, Doll R, Buckley JD, Sporn MB. Can Dietary Beta-carotene M aterially Reduce Cancer Rates? Nature 1981; 290: 201-8.

12. Gaby SK, Bendich A, Singh VN, M achlin LJ (eds.) Vitamin Intake and Health. New York: M arcel Dekker 1991; 17-27.

13. Rui Hai Liu. Potential Synergy of Phytochemicals in Cancer Prevention: Mechanism of Action. J Nutr 2004; 134: 34795-855

14. Kaugras EG, Silverman S, Lovas JGL, Thompson JS, Brandt RB, Singh VN. Role of Antioxidant Supplements in the Treatment of Human Oral Leukoplakia. Oral Surgery Oral M edicine Oral Pathology 1996; 81: 514.

15. Agarwal S, Rao AV. Tomato Lycopene and Low Protein Lipoprotein Oxidation: A Human Dietary Intervention Study. Lipids 1998; 33: 981 4.

16. Matsushima NR, Shidoji Y, Nishiwaki S, Yamada T, M oriwaki H, M uto Y Suppression by carotenoids of microcystin-induced morphological changes in mouse hepatocytes. Lipids 1995; 30:1029-34.

17. Levy J, Bosin E, Feldman B, Giat Y, M iinster A, Danilenko M, Sharoni Y.
Lycopene is a M ore Potent Inhibitor of Human Cancer Cell Proliferation than Either Alpha-carotene or Beta-carotene. Nutr Cancer 1995; 24:257-66.

18. Sunderraj S, Sharma R, Agarwal V, Narang P, Reddy YG, Sharma AK. An In vivo Study to Determine the Efficacy of Lycopene as Compared to Multi-vitamin Preparation in the Treatment of Oral Sumucus Fibrosis. J lindian Aca Oral M ed Radiol 2012; 24: 190-3.

19. Nijveldt RJ, van Nood E, van Hoorn DEC, Boelens PG, van Norren K, van Leeuwen PAM . Flavonoids: A Review of Probable M echanism of Action and Potential Applications. AmJ Clin Nutr 2001; 74: 418-25.

20. Korkina LG, Afanas'ev IB. Antioxidant and Chelating Properties of Flavonoids. Adv Pharmacol 1997; 38: 151-63.

21. van Acker SA, Tromp MN, Haenen GR, van der Vijgh WJ, Bast A. Flavonoids as Scavengers of Nitric Oxide Radical. Biochem Biophys Res Commun 1995; 214: 755-9

22. Chang WS, Lee YJ, Lu FJ, Chiang HC. Inhibitory Effects of Flavonoids on Xanthine Oxidase. Anticancer Res 1993; 13: 2165-70.

23. Bennett JP, Gomperts BD, Wollenweber E. Inhibitory Effects of Natural Flavonoids on Secretion from Mast Cells and Neutrophils. Arzneimittelforschung 1981; 31: 433-7.

24. de Ghroot H, Rauen U. Tissue Injury by Reactive Oxygen Species and the Protective Effects of Flavonoids. Fundam Clin Pharmacol 1998; 12: 249-55.

25. Dragsted LO, Strube M, Larsen JC. Cancer-protective Factors in Fruits and Vegetables: Biochemical and Biological Background. Pharmacol Toxicol 1993; 72: 116-35.

26. Waladkhani AR, Clemens MR. Effect of Dietary Phytochemicals on Cancer Development. Int J M ol M ed 1998; 1: 747-53.

27. Shetti A, Keluskar V, Aggarwal A. Antioxidants: Enhancing Oral and General Health. JIAOM R 2009; 21: 1-6.

28. Kaugras EG, Silverman S, Lovas JGL. A Clinical Trial of Antioxidant Supplements in the Treatment of Oral Leukoplakia. Oral Surg Oral M ed Oral path 1994; 78: 462-8.

29. Mathews-Roth MM. Beta-carotene: Clinical Aspects. In: New Protective Roles for Selected Nutrients. Alan R Liss 1989; 17-38.

30. Stich HF, Rosin MP, Hornby AP. Remission of Oral Leukoplakias and Micronuclei in the Tobacco/betel Nut Quid Chewers Treated with Beta-carotene and with Beta-carotene plus Vitamin A. Int J Cancer 1988; 42: 195-9.

31. Benner SE', Winn RJ, Lippman SM , Poland J, Hansen KS, Luna M A, Hong WK. Regression of Oral Leukoplakia with Alpha-tocopherol: A Community Clinical Oncology Program Chemoprevention Study. J Natl Cancer Inst 1993; 85: 44-7.

32. Singh M, Krishnappa R, Bagewadi A, Keluskar V. Efficacy of Oral Lycopenein the Treatment of Oral Leukoplakia. Oral Oncology 2004; 40: 591-6. 